\title{
Surface Waves Along a Perfectly Conducting Plane Covered With Semi-Infinite Magneto-Plasma
}

\author{
S. Adachi and Y. Mushiake \\ Contribution From the Department of Electrical Communications, Tohoku University, Sendai, Japan \\ (Received August 31, 1964)

\begin{abstract}
The electromagnetic fields in the semi-infinite magneto-plasma bounded by the perfectly conducting plane in the presence of the infinite electric line current of an arbitrary phase constant are derived in integral forms using Fourier transform method. The propagation characteristics of the possible surface waves excited along the conducting plane in arbitrary directions with respect to the direction of magnetization are discussed in detail.
\end{abstract}

\section{Introduction}

Among a number of boundary value problems on electromagnetic waves in media containing magnetoplasmas, one of the simplest and most fundamental problems would be the wave propagation in the semiinfinite magneto-plasma bounded by a perfectly conducting plane. Regarding this problem Ishimaru [1962] and Seshadri [1962] have shown that the perfectly conducting plane can support a surface wave when the impressed $\mathrm{d}-\mathrm{c}$ magnetic field is parallel to the plane and is transverse to the direction of propagation. In this particular case the surface wave is transverse electric and magnetic, and has a unidirectional property. This analysis has been extended to an inhomogeneous plasma recently by Wait [1964].

The principal purpose of this paper is to study the characteristics of the surface wave propagating in arbitrary directions with respect to the d-c magnetic field. Unless the plasma is magnetized transversely to the direction of propagation the electromagnetic fields can not be separated into TE and TM modes, and are expressed by a combination of two characteristic plane wave modes, each having different, but inter-related characteristic values.

In section 2 the electromagnetic fields due to an infinite line current being transverse to the $\mathrm{d}$-c magnetic field and having an arbitrary phase constant are derived in integral forms using Fourier transform method. Section 3 discusses exclusively the characteristics of the possible surface waves excited along the conducting plane in arbitrary directions when the phase constant of the line current is assumed to take an arbitrary value.

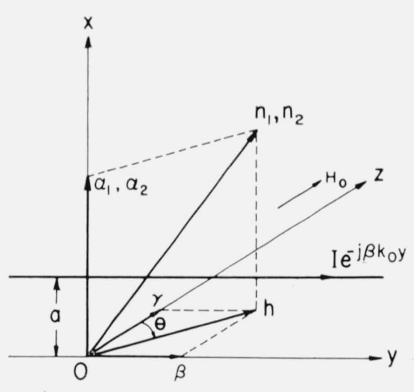

FIgURE 1. Coordinate system and notations.

\section{Integral Solutions of Electromagnetic Fields Due to a Line Current}

In order to consider surface waves propagating in arbitrary directions with respect to the d-c magnetic field an infinite line current of amplitude $I$ parallel to the conducting plane is assumed to have an arbitrary phase constant, $k_{0} \beta$ ( $\beta$ : relative phase constant with respect to a free space phase constant $k_{0}$ ). The coordinate is chosen so that the perfectly conducting plane is located in the $y-z$ plane $(x=0)$ and the line current is in the direction of $y \quad(z=0)$ with a height $a$ above the plane as shown in figure 1 . The semi-infinite lossless plasma $(x \geq 0)$ is magnetized in the $z$-direction. 
Maxwell's equations for the region of the semiinfinite lossless magneto-plasma lead to

$$
\left(\nabla \nabla-\nabla^{2} \hat{\hat{\mathbf{I}}}-k_{0}^{2 \hat{\hat{\epsilon}})} \vec{E}=-j \omega \mu_{0} I e^{-j \beta k_{0} y} \delta(x-a) \delta(z) \vec{y},\right.
$$

where $\hat{\hat{\mathbf{I}}}$ is a unit tensor and $\hat{\hat{\boldsymbol{\epsilon}}}$ is the relative dielectric tensor expressed by the matrix:

$$
\begin{gathered}
\hat{\hat{\epsilon}}=\left(\begin{array}{ccc}
\epsilon_{1} & -j \epsilon_{2} & 0 \\
j \epsilon_{2} & \epsilon_{1} & 0 \\
0 & 0 & \epsilon_{3}
\end{array}\right), \\
\epsilon_{1}=1-\frac{\omega_{p}^{2}}{\omega^{2}-\omega_{H}^{2}}, \epsilon_{2}=\frac{\omega_{H}}{\omega} \frac{\omega_{p}^{2}}{\omega^{2}-\omega_{H}^{2}}, \epsilon_{3}=1-\frac{\omega_{p}^{2}}{\omega^{2}},
\end{gathered}
$$

where $\omega, \omega_{p}$, and $\omega_{H}$ are the frequency, the electron plasma frequency, and the electron gyrofrequency, respectively. All the field components would be assumed to have the time and the $y$ dependence of $e^{j \omega t-j \beta k_{0} y}$, which leads to the substitution, $\partial / \partial y=$ $-j \beta k_{0}$ and $\partial^{2} / \partial^{2} y=-\beta^{2} k_{0}^{2}$. Let the relative propagation constants in the direction of $x$ and $z$ be denoted by $\alpha$ and $\gamma$, respectively. Applying the double Fourier transformation to (1) one obtains

$$
\begin{aligned}
& \left(\begin{array}{ccc}
\beta^{2}+\gamma^{2}-\epsilon_{1} & -\alpha \beta+j \epsilon_{2} & -\alpha \gamma \\
-\alpha \beta-j \epsilon_{2} & \alpha^{2}+\gamma^{2}-\epsilon_{1} & -\beta \gamma \\
-\alpha \gamma & -\beta \gamma & \alpha^{2}+\beta^{2}-\epsilon_{3}
\end{array}\right) \overline{\overline{\mathbf{E}}} \\
& =-\frac{j \omega \mu_{0} I}{2 \pi} e^{j \alpha k_{0} a}\left(\begin{array}{l}
0 \\
1 \\
0
\end{array}\right),
\end{aligned}
$$

where the proper behavior of $\vec{E}$ at infinity is assumed.

Solving (4) with respect $\overline{\bar{E}}$, one obtains the transforms of the primary electric fields due to the line current. For example $\overline{\bar{E}}_{y}^{(P)}$ is given by

$$
\overline{\bar{E}}_{y}^{(P)}=j \frac{I e^{j \alpha k_{0} a}}{2 \pi \omega \epsilon_{0} \epsilon_{1}} \cdot \frac{\alpha^{2}\left(\beta^{2}-\epsilon_{1}\right)+\left(h^{2}-\epsilon_{1}\right)\left(\beta^{2}-\epsilon_{3}\right)}{\left(\alpha^{2}-\alpha_{1}^{2}\right)\left(\alpha^{2}-\alpha_{2}^{2}\right)},
$$

where

$$
h^{2}=\beta^{2}+\gamma^{2}
$$

and $\alpha_{1}^{2}, \alpha_{2}^{2}$ are the solutions of the quadratic equation with respect to $\alpha^{2}$ :

$$
\begin{aligned}
\epsilon_{1} \alpha^{4}+\left[\epsilon_{1}\left(h^{2}+\beta^{2}\right)+\epsilon_{3} \gamma^{2}-\epsilon_{1}^{2}+\epsilon_{2}^{2}-\epsilon_{1} \epsilon_{3}\right] \alpha^{2} \\
+\left(h^{2}-\epsilon_{1}\right)\left[\epsilon_{1}\left(\beta^{2}-\epsilon_{3}\right)+\epsilon_{3} \gamma^{2}\right]+\epsilon_{2}^{2}\left(\beta^{2}-\epsilon_{3}\right)=0,
\end{aligned}
$$

which one obtains by equating the determinant of the matrix in the left hand side of (4) to zero. These two characteristic values correspond to the relative propagation constants in the $x$-direction of the ordi- nary and/or extraordinary waves propagating in an infinite magneto-plasma medium whose relative propagation constants are denoted by $n_{1}$ and $n_{2}$ in figure 1.

The primary electric fields are given by the inverse transform of $\overline{\bar{E}}$ with respect to $\alpha$ and $\gamma$. The integration with respect to $\alpha$ can be carried out by obtaining the residues of the integrands at $\alpha=\alpha_{1}, \alpha_{2}$ or $-\alpha_{1},-\alpha_{2}$ where the signs of $\alpha_{1}$ and $\alpha_{2}$ are chosen such that $\mathscr{I}_{m}\left(\alpha_{1}, \alpha_{2}\right)<0$ (fig. $2 \mathrm{a}$ and $2 \mathrm{~b}$ ). The resulting primary electric fields are given by

$$
\begin{aligned}
& E_{x}^{(P)}=-K \int_{-\infty}^{\infty}\left[\frac{X\left( \pm \alpha_{1}\right)}{\alpha_{1}\left(\alpha_{1}^{2}-\alpha_{2}^{2}\right)} e^{-j \alpha_{1} k_{0}|x-a|}\right. \\
& \left.\quad-\frac{X\left( \pm \alpha_{2}\right)}{\alpha_{2}\left(\alpha_{1}^{2}-\alpha_{2}^{2}\right)} e^{-j \alpha_{2} k_{0}|x-a|}\right] e^{-j \gamma k_{0} z} d \gamma \quad x \lessgtr a, \\
& E_{y}^{(P)}=K \int_{-\infty}^{\infty}\left[\frac{Y\left(\alpha_{1}\right)}{\alpha_{1}\left(\alpha_{1}^{2}-\alpha_{2}^{2}\right)} e^{-j \alpha_{1} k_{0}|x-a|}\right. \\
& \left.\quad-\frac{Y\left(\alpha_{2}\right)}{\alpha_{2}\left(\alpha_{1}^{2}-\alpha_{2}^{2}\right)} e^{-j \alpha_{2} k_{0}|x-a|}\right] e^{-j \gamma k_{0} 2} d \gamma,
\end{aligned}
$$

$E_{z}^{(P)}=k_{0} K \int_{-\infty}^{\infty}\left[\frac{Z\left( \pm \alpha_{1}\right)}{\alpha_{1}\left(\alpha_{1}^{2}-\alpha_{2}^{2}\right)} e^{-j \alpha_{1} k_{0} \mid x-a !}\right.$

$$
\left.-\frac{Z\left( \pm \alpha_{2}\right)}{\alpha_{2}\left(\alpha_{1}^{2}-\alpha_{2}^{2}\right)} e^{-j \alpha_{2} k_{0}|x-a|}\right] \gamma e^{-j \gamma k_{0} z} d \gamma \quad x \lessgtr a,
$$

where

$$
\begin{aligned}
X(\alpha) & =\left(\alpha \beta+j \epsilon_{2}\right)\left(\alpha^{2}+\beta^{2}-\epsilon_{3}\right)+\alpha \beta \gamma^{2}, \\
Y(\alpha) & =\alpha^{2}\left(\beta^{2}-\epsilon_{1}\right)+\left(h^{2}-\epsilon_{1}\right)\left(\beta^{2}-\epsilon_{3}\right), \\
Z(\alpha) & =\alpha\left(\alpha \beta+j \epsilon_{2}\right)+\beta\left(h^{2}-\epsilon_{1}\right), \\
K & =\sqrt{\frac{\mu_{0}}{\epsilon_{0}}} \frac{I e^{-j \beta k_{0} y}}{4 \pi \epsilon_{1}} .
\end{aligned}
$$

The secondary waves to be added to the primary waves in order that the tangential electric fields vanish at the perfectly conducting plane are obtained as a solution of the homogeneous wave equation
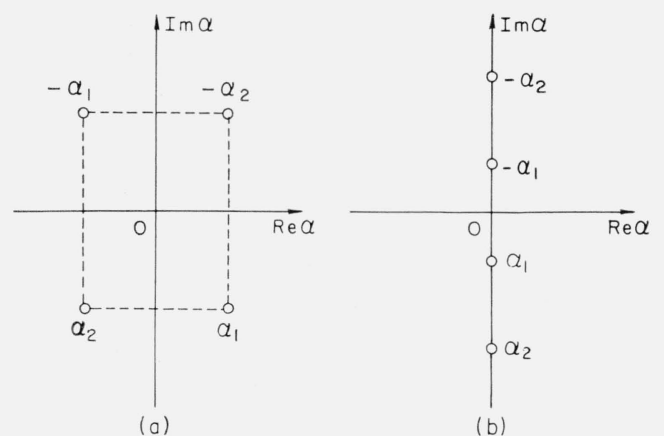

Figure 2a, b. Poles in the a plane for real values of $\mathrm{h}^{2}$. 
corresponding to (1); i.e.,

$$
\begin{aligned}
E_{x}^{(S)}=-K \int_{-\infty}^{\infty}\left[A \frac{X\left(-\alpha_{1}\right)}{Y\left(\alpha_{1}\right)} e^{-j \alpha_{1} k_{0} x}\right. & \\
& \left.+B \frac{X\left(-\alpha_{2}\right)}{Y\left(\alpha_{2}\right)} e^{-j \alpha_{2} k_{0} x}\right] e^{-j \gamma k_{0} z} d \gamma,
\end{aligned}
$$

$E_{y}^{(S)}=K \int_{-\infty}^{\infty}\left[A e^{-j \alpha_{1} k_{0} x}+B e^{-j \alpha_{2} k_{0} x} e^{-j \gamma k_{0} z} d \gamma\right]$,

$E_{z}^{(S)}=k_{0} K \int_{-\infty}^{\infty}\left[A \frac{Z\left(-\alpha_{1}\right)}{Y\left(\alpha_{1}\right)} e^{-j_{\alpha_{1}} k_{0} x}\right.$

$$
\left.+B \frac{Z\left(-\alpha_{2}\right)}{Y\left(\alpha_{2}\right)} e^{-j \alpha_{2} k_{0} x}\right] \gamma e^{-j \gamma k_{0} z} d \gamma
$$

Introducing the boundary conditions leads to

$A=Y\left(\alpha_{1}\right)$

$\times \frac{\left(\alpha_{1}+\alpha_{2}\right) P\left(-\alpha_{1}, \alpha_{2}, \beta, \gamma\right) e^{-j \alpha_{1} k_{0} a}+j 2 \epsilon_{2} \alpha_{1} Y\left(\alpha_{2}\right) e^{-j \alpha_{2} k_{0} a}}{\alpha_{1}\left(\alpha_{1}-\alpha_{2}\right)\left(\alpha_{1}^{2}-\alpha_{2}^{2}\right) P\left(\alpha_{1}, \alpha_{2}, \beta, \gamma\right)}$,

$B=Y\left(\alpha_{2}\right)$

$\times \frac{j 2 \epsilon_{2} \alpha_{2} Y\left(\alpha_{1}\right) e^{-j \alpha_{1} k_{0} a}+\left(\alpha_{1}+\alpha_{2}\right) P\left(\alpha_{1},-\alpha_{2}, \beta, \gamma\right) e^{-j \alpha_{2} k_{0} a}}{\alpha_{2}\left(\alpha_{1}-\alpha_{2}\right)\left(\alpha_{1}^{2}-\alpha_{2}^{2}\right) P\left(\alpha_{1}, \alpha_{2}, \beta, \gamma\right)}$,

where

$$
\begin{aligned}
P\left(\alpha_{1}, \alpha_{2}, \beta, \gamma\right)= & \left(h^{2}-\epsilon_{1}\right)\left\{\beta\left(\epsilon_{1}-\epsilon_{3}\right)\left(\alpha_{1}+\alpha_{2}\right)\right. \\
& \left.-j \epsilon_{2}\left(\beta^{2}-\epsilon_{3}\right)\right\}+j \epsilon_{2}\left(\beta^{2}-\epsilon_{1}\right) \alpha_{1} \alpha_{2} .
\end{aligned}
$$

\section{Surface Waves in Arbitrary Directions}

In order to discuss the possible surface waves which may propagate in arbitrary directions with respect to the $\mathrm{d}$-c magnetic field, let $\beta$ take arbitrary values of $-\infty<\beta<\infty$; let $\beta$ and $\gamma$ in (7) and (12) be expressed by

$$
\left.\begin{array}{l}
\beta=h \sin \theta \\
\gamma=h \cos \theta
\end{array}\right\} .
$$

The propagation constant $h$ of the possible surface wave propagating in an arbitrary direction $\theta$ must simultaneously satisfy (7) and the equation

$$
P\left(\alpha_{1}, \alpha_{2}, h, \theta\right)=0,
$$

which is obtained from the denominator of (11). For the later use an alternative expression of (14) with (12) is derived from (7) and (14) as follows:

$$
\left(\gamma^{2}-\epsilon_{1}\right)\left(h^{2}-\epsilon_{1}-\alpha_{1} \alpha_{2}\right)+j \epsilon_{2} \beta\left(\alpha_{1}+\alpha_{2}\right)-\epsilon_{2}^{2}=0 .
$$

It does not seem feasible unfortunately to solve (7) and (14) [or (14a)] for $h$ explicitly. First con- sider the special cases of $\theta=0, \pi$, and $\pm \pi / 2$. When $\theta$ is equal to 0 or $\pi, h^{2}$ and $\alpha_{1,2}^{2}$ can be obtained explicitly [Adachi and Mushiake, 1964] as

$$
h^{2}=\epsilon_{1} \pm \sqrt{\epsilon_{1}\left(\epsilon_{1}-1\right)},
$$

$\alpha_{1,2}^{2}=-\frac{1}{2 \epsilon_{1}}\left\{\left(\epsilon_{1}+\epsilon_{3}\right)\left(h^{2}-\epsilon_{1}\right)\right.$

$$
\left.+\epsilon_{2}^{2} \pm \epsilon_{2} \sqrt{2\left(\epsilon_{1}+\epsilon_{3}\right) h^{2}-\epsilon_{1}+\epsilon_{3}}\right\} \cdot
$$

For $\omega$ smaller than $\left(\omega_{p}^{2}+\omega_{H}^{2}\right)^{1 / 2}, h^{2}$ given by (15) is always real, and $\alpha_{1} \alpha_{2}$ also is real from (7) and (12). For this case the signs $( \pm)$ in (15) correspond to $\epsilon_{1} \epsilon_{3} \lesseqgtr 0$ if $\alpha_{1} \alpha_{2}<0$, and $\epsilon_{1} \epsilon_{3} \gtrless 0$ if $\alpha_{1} \alpha_{2}>0$, respectively. When $\alpha_{1} \alpha_{2}$ is positive, imaginary parts of both $\alpha_{1}$ and $\alpha_{2}$ cannot be negative simultaneously, therefore the fields increase to infinity when $x \rightarrow \infty$.

When $\alpha_{1} \alpha_{2}$ is negative, and in the frequency range of

$$
\omega_{p}<\omega<\sqrt{\omega_{p}^{2}+\omega_{H}^{2}},
$$

either $\alpha_{1}$ or $\alpha_{2}$ is a positive real and the other is a negative real. Therefore no surface waves are excited in this frequency region.

Finally in the frequency range of

$$
\omega<\operatorname{Min} .\left(\omega_{H}, \omega_{p}\right) \text {, }
$$

both $\alpha_{1}$ and $\alpha_{2}$ have negative imaginary parts, and as a result the slow waves are trapped along the conducting plane. Thus the relative propagation constant $h$ is given by $\left.\left(\epsilon_{1}+\sqrt{\epsilon_{1}\left(\epsilon_{1}-1\right.}\right)\right)^{1 / 2}>1$ and is plotted by the solid line in figures $3 \mathrm{a}$ and $3 \mathrm{~b}$, where the dashed line shows $h$ of the improper mode whose amplitude increases to infinity when $x \rightarrow \infty$. The group velocity $v_{g}$ of this trapped surface wave is obtained as

$$
c / v_{g}=h\left[1+\left(1-\frac{1}{2 h^{2}}\right) \frac{\omega_{p} \omega^{2}}{\left(\omega_{H}^{2}-\omega^{2}\right)\left(\omega_{p}^{2}+\omega_{H}^{2}-\omega^{2}\right)^{1 / 2}}\right],
$$

where $c$ is the velocity of light.

Next consider the special cases of $\theta= \pm \pi / 2$. Obviously $h^{2}=\epsilon_{1}$ is a solution of (14). From (14a) and $(7)$ one obtains

$$
\begin{array}{r}
h=\sqrt{\epsilon_{1}} \\
\alpha_{1}=\mp j \frac{\epsilon_{2}}{\sqrt{\epsilon_{1}}}, \alpha_{2}= \pm j \sqrt{\epsilon_{1}-\epsilon_{3}}, \\
\\
\end{array}
$$

In this particular angle of $\theta$, only $E_{x}$ and $H_{z}$ remain finite: the surface waves are completely transverse electric and magnetic. At $\theta=-\pi / 2$, if the frequency is below the gyrofrequency $\omega_{H}$, both $\alpha_{1}$ and $\alpha_{2}$ are negative imaginaries $\left(\alpha_{2}\right.$ is in fact independent on the TEM surface wave), hence the slow surface wave 

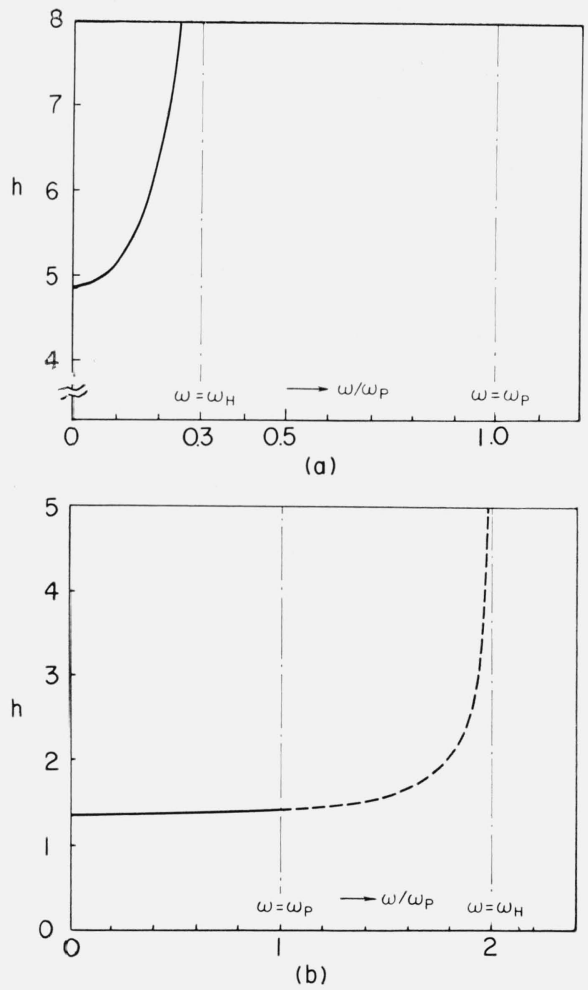

FiguRE 3a, b. Frequency characteristics of the relative propagation constant, h of the trapped surface wave propagating in the direction of $d-c$ magnetic field.

(a): $\omega_{H}<\omega_{p}$; (b): $\omega_{H}>\omega_{p}$. The dashed curve shows that for the impropr mode

(h>1) is trapped along the conductor. At $\theta=\pi / 2$, if the frequency is larger than the frequency $\sqrt{\omega_{p}^{2}+\omega_{H}^{2}}$, $h$ is a positive real less than 1 and $\alpha_{1}$ is a negative imaginary. This type of wave is not completely trapped if $\theta \neq \pi / 2$ because of the real value of $\alpha_{2}$. Thus the fast surface wave is trapped only in the direction of $\theta=\pi / 2$. This is the unidirectional surface wave given by Ishimaru [1962] and Seshadri [1962].

The relative phase constant $h$ for arbitrary directions are calculated numerically. Figure 4 shows the phase velocity surface for $\omega_{H} / \omega_{p}=0.3, \omega / \omega_{p}=0.1$, and 0.2 . The phase velocity surface is asymmetrical with respect to the direction of d-c magnetic field due to the presence of the term $\beta(=h \sin \theta)$ in (12). The ratio of the phase velocity at $\theta=0, \pi$ to that at $\theta=-\pi / 2$ is found to take a value between one and two in all cases since $\epsilon_{1}$ varies in the range of $1<\epsilon_{1}<\infty$ if $\omega<\omega_{H}$.

At a certain angle denoted by $\theta_{c}$ and $\left(\pi-\theta_{c}\right)$ in figure 4 , $\mathscr{I} m\left(\alpha_{1}\right.$ and/or $\left.\alpha_{2}\right)$ becomes zero. Beyond this critical angle any real values of $h^{2}$ can not satisfy the simultaneous equations (7) and (14). Consequently the trapped surface wave transforms to the leaky surface wave whose propagation constants are complex when $\theta$ crosses this critical angle.

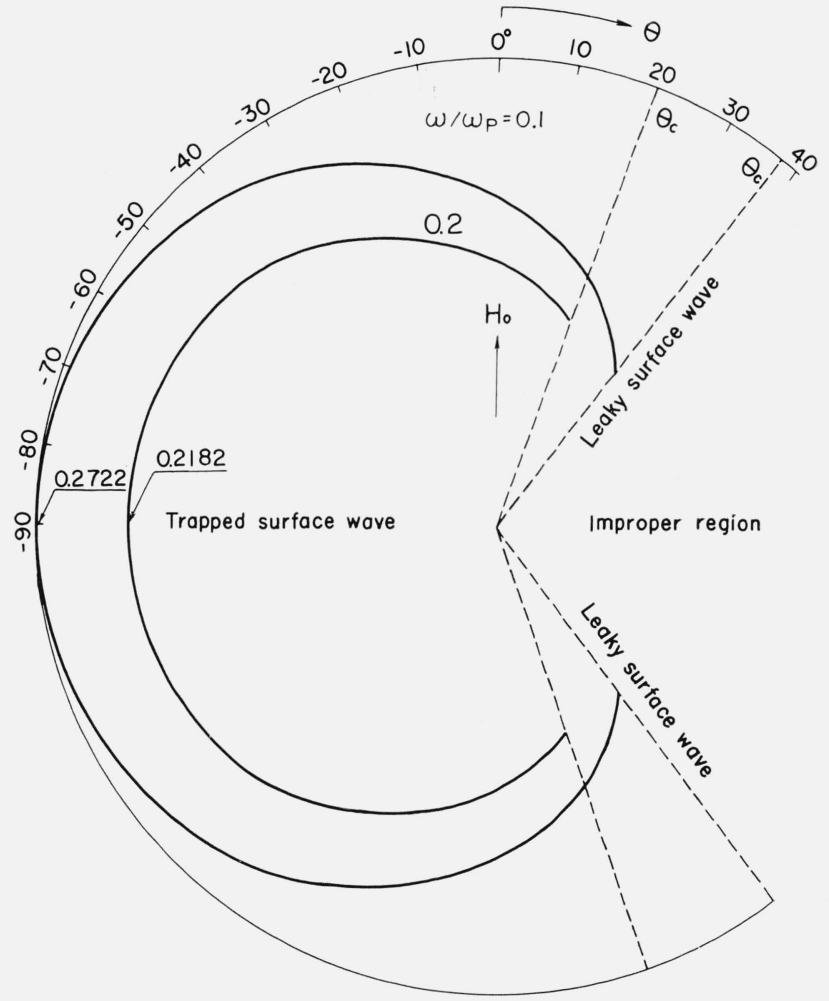

FIGURE 4. Phase velocity surface $(1 / \mathrm{h})$ for $\omega_{H} / \omega_{p}=0.3$.

At $\theta=\pi / 2, \alpha_{1}$ and $\alpha_{2}$ become both positive imaginaries, leading to an improper mode with a real $h$ as obtained in $(20)$.

In the region of the trapped surface wave, $-\left(\pi+\theta_{c}\right)<\theta<\theta_{c}, j \alpha_{1}$ and $j \alpha_{2}$ are both real or complex conjugate with positive real parts, hence both $A$ and $B$ in (11) also are pure imaginary or complex conjugate, respectively. As a result the equiphase surface is found to be always vertical to the guiding plane.

\section{Conclusion}

In section 2 the electromagnetic fields in the semiinfinite magnetoplasma bounded by the perfectly conducting plane in the presence of the infinite electric line current were first derived in integral forms using Fourier transform method. The propagation characteristics of the possible surface waves excited along the conducting plane in arbitrary directions were discussed in section 3 , where the phase constant of the line current was assumed to take an arbitrary value.

It is concluded that the slow surface wave is trapped along the perfectly conducting plane when the frequency is less than the gyrofrequency, and the 
propagating direction is in the region of $-\left(\pi+\theta_{c}\right)$ $<\theta<\theta_{c}$, beyond which the trapped surface wave transforms to the leaky surface wave. Besides the above surface wave the unidirectional fast TEM surface wave exists in the particular direction of $\theta=\pi / 2$ when the frequency is larger than $\sqrt{\omega_{p}^{2}+\omega_{H}^{2}}$ as already pointed out by Ishimaru [1962] and Seshadri [1962].

The electromagnetic fields due to the infinite magnetic line current have been obtained quite similarly with those due to the electric line current. The expressions for $P\left(\alpha_{1}, \alpha_{2}, \beta, \gamma\right)$ are the same in both cases. Consequently all of the discussions in section 3 hold also for the case of the magnetic current excitation as they stand.

\section{References}

Adachi, S., and Y. Mushiake (1964), Surface wave on a perfectly conducting plane covered with magnetoplasma, IEEE Trans. Microwave Theory Tech. MTT 12, No. 5 (communications).

Ishimaru, A. (1962), The effect of a unidirectional surface wave along a perfectly conducting plane on the radiation from a plasma sheath, presented at the Second Symp. on the Plasma Sheath, Boston, Mass.

Seshadri, S. R. (1962), Excitation of surface waves on a perfectly conducting screen covered with anisotropic plasma, IRE Trans. Microwave Theory Tech. MTT 10, $573-578$

Wait, J. R. (1964), Concerning the theory of radiation from a slotted conducting plane in a plasma environment, NBS Tech. Note 223.

(Paper 69D2-451) 